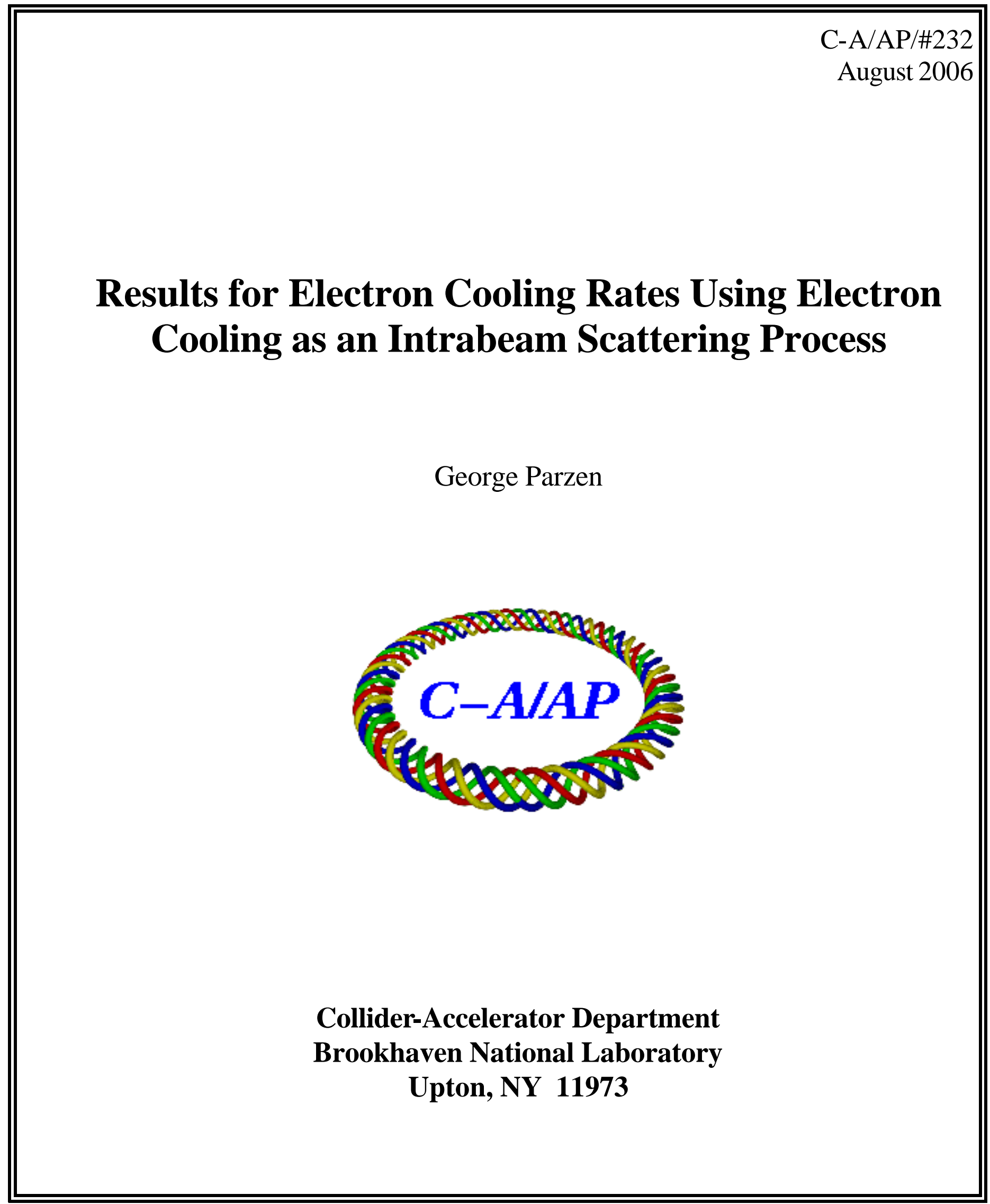




\title{
Recults for electron cooling rates using electron cooling as an intrabeam scattering process
}

\author{
George Parzen
}

August 18, 2006

\begin{abstract}
Electron cooling that results when a bunch of electrons overlaps a bunch of ions, with both bunches moving at the same velocity, may be considered to be an intrabeam scattering process. This paper lists the results found for the emittance cooling rates using the methods developed in intrabeam scattering theory. Only the results are given here. Derivations of these results will be given in a future paper. It is assumed that the dispersion is zero in the cooling section, and that the ion bunch and the electron bunch have gaussian distributions.
\end{abstract}

\section{Introduction}

Electron cooling that results when a bunch of electrons overlaps a bunch of ions, with both bunches moving at the same velocity, may be considered to be an intrabeam scattering process. The process is similar to the usual intrabeam scattering where the ions scatter from each other and usually results in beam growth. An important difference is that in electron cooling the mass of the ion is different from and much larger than the mass of the electron. This difference considerably complicates the intrabeam scattering theory. It introduces a new term in the emittance growth rate, which vanishes when the particles are identical and their masses are equal, and can give rise to emittance cooling of the heavier particles. The term that gives rise to beam growth for the usual intrabeam scattering is also present but is much smaller than the cooling term when one particle is much heavier than the other. 
This paper lists the results found for the emittance cooling rates due to the scattering of the ions in the ion bunch by the electrons in the electron bunch. Only the results are given here. Derivations of these results will be given in a future paper, Ref.[3]. It is assumed that the dispersion is zero in the cooling section, that the ion bunch and the electron bunch have gaussian distributions, and that there are no magnetic fields in the cooling section.

\section{Results}

Ions are indicated by the subscrpt a and the electrons by the subscript $\mathrm{b}$. The cooling rate for $\left\langle p_{i a}^{2} / p_{0 a}^{2}\right\rangle, \quad i=x, s, y$ in the Rest CS is due to the scattering of the ions from the electrons. This cooling rate has to be added to the growth rate due to the scattering of the ions from each other to find the actual growth rate of the ions. $p_{0 a}$ is the central momentun of the ion bunch. The assumed gaussian distributions of the bunches are described by the parameters

$$
\bar{\epsilon}_{i a}, \beta_{i a}, \alpha_{i a}, \quad \bar{\epsilon}_{i b}, \beta_{i b}, \alpha_{i b}, \quad i=x, s, y
$$

The gaussian distribution is further defined using these parameters in Ref.[1].

\section{$2.1 d<p_{i a} p_{j a}>/ d t, \quad i, j=x, s, y$ in the Rest CS.}

$$
\begin{aligned}
\delta<\left(\bar{p}_{i a} \bar{p}_{j a}\right)>= & \frac{N_{b}}{\Gamma_{a} \Gamma_{b}} \frac{1}{\bar{A}_{p}^{1 / 2}} \pi^{3} r_{a b}^{2} c \frac{\mu}{m_{a}} \hat{W}_{i j} \\
& \int d^{3} \Delta \frac{\exp \left[-\left(\lambda_{x} \Delta_{x}^{2}+\lambda_{y} \Delta_{y}^{2}+\lambda_{s} \Delta_{s}^{2}\right)\right]}{\beta_{a b}^{3}} \Delta_{i} \Delta_{j} \\
& \ln \left[1+\left(\frac{\beta_{a b}^{2} b_{\text {maxab }}}{r_{a b}}\right)^{2}\right] d t \\
\hat{W}_{i j}= & 2 \pi\left[\left(W_{\eta} \frac{B_{10}}{2}+W_{p_{\eta}} \frac{B_{01}}{2}\right)_{i}+\left(W_{\eta} \frac{B_{10}}{2}+W_{p_{\eta}} \frac{B_{01}}{2}\right)_{j}\right] \\
\beta_{a b}= & \gamma_{0} \beta_{0}\left(\Delta_{x}^{2}+\Delta_{y}^{2}+\Delta_{s}^{2}\right)^{1 / 2} \\
\lambda_{i}= & {\left[A_{00}-\left(\frac{B_{10}}{2}\right)^{2}-\left(\frac{B_{01}}{2}\right)^{2}\right]_{i} } \\
\bar{A}_{i}= & {\left[A_{11} A_{22}-A_{12}^{2}\right]_{i} }
\end{aligned}
$$




$$
\begin{aligned}
\bar{A}_{p}^{1 / 2} & =\bar{A}_{x}^{1 / 2} \bar{A}_{y}^{1 / 2} \bar{A}_{s}^{1 / 2} \\
x_{\eta i} & =\left[\frac{A_{22}^{1 / 2}}{\bar{A}^{1 / 2}}\right]_{i} \quad W_{\eta i}=\left[-\frac{A_{12}}{\bar{A}^{1 / 2}}\right]_{i} \quad W_{p_{\eta i}}=\left[\frac{1}{A_{22}^{1 / 2}}\right]_{i} \\
B_{10 i} & =\left[A_{10} x_{\eta}+A_{01} W_{\eta}\right]_{i} \quad B_{01 i}=\left[A_{01} W_{p_{\eta}}\right]_{i} \\
B_{10 i} & =\left[A_{10} \frac{A_{22}^{1 / 2}}{\bar{A}^{1 / 2}}-A_{01} \frac{A_{12}}{\bar{A}^{1 / 2}}\right]_{i} \quad B_{01 i}=\left[A_{01} \frac{1}{A_{22}^{1 / 2}}\right]_{i} \\
A_{11 i} & =\left[\frac{1+\alpha_{i}^{2}}{\beta_{i} \bar{\epsilon}_{i}}\right]_{+} \quad A_{22 i}=\left[\frac{\beta_{i}}{\bar{\epsilon}_{i}}\right]_{+} \\
A_{12 i} & =\left[\frac{\alpha_{i}}{\bar{\epsilon}_{i}}\right]_{+} \quad A_{10 i}=\left[2 \frac{\mu}{m} \frac{\alpha_{i}}{\bar{\epsilon}_{i}}\right]_{-} \\
A_{01 i} & =\left[2 \frac{\mu}{m} \frac{\beta_{i}}{\bar{\epsilon}_{i}}\right]_{-} \quad A_{00 i}=\left[\left(\frac{\mu}{m}\right)^{2} \frac{\beta_{i}}{\bar{\epsilon}_{i}}\right]_{+}
\end{aligned}
$$

$b_{\text {maxab }}$ is the largest allowed impact parameter for the scattering of the ions by the electrons. $\beta_{s}$ in the Rest CS is larger than $\beta_{s}$ in the Laboratory CS by the factor $\gamma_{0}^{2} . s, p_{s}$ are the particle longitudinal position and momentum in the Rest CS.

The symbols $[()]_{+}$and $[(\quad)]_{-}$are defined by

$$
\begin{aligned}
& {[(\quad)]_{+}=()_{a}+()_{b}} \\
& {[()]_{-}=()_{a}-()_{b}}
\end{aligned}
$$

The integral over $d^{3} \Delta$ is an integral over all possible values of the relative velocity of any two particles in a bunch. $\beta_{0}, \gamma_{0}$ are the beta and gamma of the center of the bunches in the Laboratory Coordinate System. $p_{0 a}$ is the momentum of the central particle in the ion bunch. With no dispersion in the cooling section, one gets zero results when $i \neq j$.

\section{2 $d<x_{i} p_{j a}>/ d t, \quad i, j=x, s, y$ in the Rest CS.}

Growth rates for the emittances require the growth rates for $\left\langle x_{i} p_{j a}\right\rangle$, $i=x, s, y$ in the Rest CS. 


$$
\begin{aligned}
\delta<\left(x_{i} \bar{p}_{j a}\right)>= & \frac{N_{b}}{\Gamma_{a} \Gamma_{b}} \frac{1}{\bar{A}_{p}^{1 / 2}} \pi^{3} r_{a b}^{2} c \frac{\mu}{m_{a}} \hat{x}_{i j} \\
& \int d^{3} \Delta \frac{\exp \left[-\left(\lambda_{x} \Delta_{x}^{2}+\lambda_{y} \Delta_{y}^{2}+\lambda_{s} \Delta_{s}^{2}\right)\right]}{\beta_{a b}^{3}} \Delta_{i} \Delta_{j} \\
& \ln \left[1+\left(\frac{\beta_{a b}^{2} b_{\text {maxab }}}{r_{a b}}\right)^{2}\right] d t \\
\hat{x}_{i j}= & 2 \pi\left[x_{\eta} \frac{B_{10}}{2}\right]_{i} \\
x_{\eta i}= & {\left[\frac{A_{22}^{1 / 2}}{\bar{A}^{1 / 2}}\right]_{i} \quad W_{\eta i}=\left[-\frac{A_{12}}{\bar{A}^{1 / 2}}\right]_{i} \quad W_{p_{\eta i}}=\left[\frac{1}{A_{22}^{1 / 2}}\right]_{i} } \\
B_{10 i}= & {\left[A_{10} x_{\eta}+A_{01} W_{\eta}\right]_{i} \quad B_{01 i}=\left[A_{01} W_{p_{\eta}}\right]_{i} } \\
B_{10 i}= & {\left[A_{10} \frac{A_{22}^{1 / 2}}{\bar{A}^{1 / 2}}-A_{01} \frac{A_{12}}{\bar{A}^{1 / 2}}\right]_{i} \quad B_{01 i}=\left[A_{01} \frac{1}{A_{22}^{1 / 2}}\right]_{i} }
\end{aligned}
$$

This expression is the same as that given for $<p_{i a} p_{j a}>$ except that $\hat{W}_{i j}$ is replaced by $\hat{x}_{i j}$.

\section{3 $d<\epsilon_{i a}>/ d t$ in the Laboratory CS $i=x, s, y$}

In the following, $d \tilde{t}$ is the time interval in the Laboratory System and $d t$ is the time interval in the Rest System. $d \tilde{t}=\gamma d t . \beta_{s}$ is the longitudinal beta function in the Laboratory System. The dispersion is assumed to be zero in the cooling section.

$$
\begin{aligned}
\frac{d}{d \tilde{t}}<\epsilon_{x a}> & =\frac{\beta_{x}}{\gamma_{0}} \frac{d}{d t}<p_{x a}^{2} / p_{0 a}^{2}>+2 \frac{\alpha_{x}}{\gamma_{0}} \frac{d}{d t}<x_{a} p_{x a} / p_{0 a}> \\
\frac{d}{d \tilde{t}}<\epsilon_{y a}> & =\frac{\beta_{y}}{\gamma_{0}} \frac{d}{d t}<p_{y a}^{2} / p_{0 a}^{2}>+2 \frac{\alpha_{y}}{\gamma_{0}} \frac{d}{d t}<y_{a} p_{y a} / p_{0 a}> \\
\frac{d}{d \tilde{t}}<\epsilon_{s a}> & =\beta_{s} \gamma_{0} \frac{d}{d t}<p_{s a}^{2} / p_{0 a}^{2}>
\end{aligned}
$$

A computer program called BIG, Ref.[2], has been written that evaluates the results given in this paper. 


\section{References}

1. G.Parzen, BNL report C-A/AP/N0.169 (2004)

2. G.Parzen, BNL report C-A/AP/N0.213 (2005)

3. G.Parzen, BNL report C-A/AP/N0.243 (2006) 